\title{
Impact of Data Exchange Provided by ATD2 Tools at Charlotte-Douglas International Airport
}

\author{
Bonny Parke ${ }^{1}$, Kimberly Jobe ${ }^{1}$, Eric Chevalley ${ }^{1}$, Savita Verma ${ }^{2}$, \\ Victoria Dulchinos ${ }^{1}$, and Lindsay Stevens ${ }^{2}$ \\ ${ }^{1}$ San Jose State University Research Foundation \\ NASA Ames Research Center \\ P. O. Box 1, Moffett Field, CA 94035-0001 \\ ${ }^{2}$ NASA Ames Research Center \\ P. O. Box 1, Moffett Field, CA 94035-0001 \\ Bonny.Parke@nasa.gov
}

\begin{abstract}
Data Exchange and Integration is necessary for progress towards an Integrated Arrival, Departure, and Surface (IADS) traffic management capability. In collaboration with the FAA, NASA has introduced new data exchange elements to Charlotte-Douglas International Airport air traffic facilities, including the American Airlines ramp, as part of the Airspace Technology Demonstration 2 (ATD2). This paper describes the new tools that deliver these elements, and the human factors impact of the tools as measured by post-bank surveys. Workload was unaffected by ATD2 tool use, and situational awareness was improved in the Tower and with Ramp controllers in the second round of surveys. Respondents described their tools as more helpful if they included ATD2 tools 1) in the Tower for insuring compliance for aircraft under a Traffic Management Initiative, 2) in the TRACON when actively used for many TRACON tasks, and 3) in the Ramp in the second round of surveys.
\end{abstract}

Keywords: Airport Surface Scheduling · Integrated Arrival, Departure, and Surface Traffic Management · Human Factors Assessment in Field Operations

\section{Introduction}

\subsection{Airspace Technology Demonstration 2 (ATD2)}

NASA has been collaborating with the Federal Aviation Administration (FAA) and aviation industry partners to develop and demonstrate new concepts and technologies for the Integrated Arrival, Departure, and Surface (IADS) traffic management capabilities under the ATD2 project. The primary goal of ATD2 is to improve the predictability and the efficiency of the air traffic system in metroplex environments while maintaining or improving throughput by integrating arrival, departure and surface prediction, scheduling, and management systems. [1] The demonstration of the tools is taking place at the Charlotte-Douglas International Airport (CLT), USA.

Data Exchange and Integration, Phase I. One of the most important underlying concepts in improving efficiency of surface operations is the sharing of data. "Data exchange and integration" was a term first coined by the FAA's Surface Collaboration Decision Making Team [2]. Other IADS concepts refer to this as "common situational 
awareness." What these terms refer to is the foundational need for surface data exchange and system-wide integration across domains, agencies and viewpoints.

In order to develop a good model of what is actually happening on the airport surface, it is necessary to understand the airport configuration and various departure scenarios from the users. Data exchange and integration includes such details as getting non-standard runway assignments when the pilot requires a longer runway for operational necessity. It includes updates on the availability of gates and good Earliest Off Block Times (EOBTs) from the carriers.

All of this data, including both electronically shared data and manually entered data, are part of the data exchange and integration functionality. This is a capability in addition to being a foundational layer that underpins the entire IADS edifice. Data exchange and integration supports surface modeling, which supports surface scheduling, which in turn enables surface metering and scheduling into the overhead stream. This capability perhaps more than any other in ATD2 is expected to be used in both nominal and off-nominal conditions, and to improve airport and National Air Space (NAS) efficiency for users every day. This capability will expand over the life of ATD2.

Data Exchange and Integration Tools in the Charlotte-Douglas Airport Facilities. In September 2017, ATD-2 tools were placed in the CLT ATC Tower, CLT TRACON, and the American Airline Ramp Tower. The Surface Trajectory Based Operations (STBO) Client tool was placed in the Tower as the primary Traffic Management Coordinator (TMC) display, and the Ramp Traffic Console (RTC) was also made accessible there. The RTC and Ramp Manager Traffic Console (RMTC) were placed in the ramp and were designed to enhance information exchange with the ATC Tower, and eventually, to provide surface metering information to the ramp controllers. The STBO Client and RTC were placed in the CLT TRACON. The STBO Client in Observer Mode was placed at the Air Route Traffic Control Center (ARTCC or Center). Before this, much of the data exchange information was conveyed via phone calls between facilities.

Human Factor Assessment of Tools. It is important to ascertain the effect of the tools and the information they conveyed to the users. Does the increase in availability of information increase their workload? Do the tools improve their situational awareness? Are airport operations seen as acceptable when using the tools? To answer these questions in a systematic way, in September 2017, surveys were administered after each of four banks (clusters of arriving and departing aircraft) per day for 5 days, after the tools were first introduced. The surveys were made available to all the users in the CLT Airport facilities: TRACON, ATC Tower, and Ramp Tower controllers and managers. A second round of surveys were administered only to ramp controllers and managers in November 2017 when they were more familiar with the tools and had increased their use of them.

This paper describes 1) some of the data exchange and integration elements, 2) the tools that conveyed these elements, 3 ) the surveys designed to elicit human factors input, and 4) the results of these surveys. 


\subsection{Data Elements Exchanged}

Some of the data elements that are currently being exchanged are listed below. Additional elements are continuously being added to this list based on user requests.

- Runway utilization

- Runway assignments

- $\quad$ Miles-in-Trail (MIT) restrictions

- Approval Requests (APREQs)/Call for Release (CFR)

- Expect Departure Clearance Times (EDCTs)

- Ground Stops at other airports

- Runway closures at CLT

- Departure fix closures

- Flight cancellations

- Gate conflicts

- Ramp closure

- Manual updates/corrections of flights

- $\quad$ Long on Board (LOB) common awareness

\subsection{STBO Client Tool}

Overview. The STBO Client provides situational awareness of surface traffic as shown below on the surface Map in Fig. 1. Also shown are the Timelines of departing and arriving aircraft on the runways to the left and right of the Map. The closer the aircraft are to the bottom of the Timelines, the sooner they will depart or arrive.

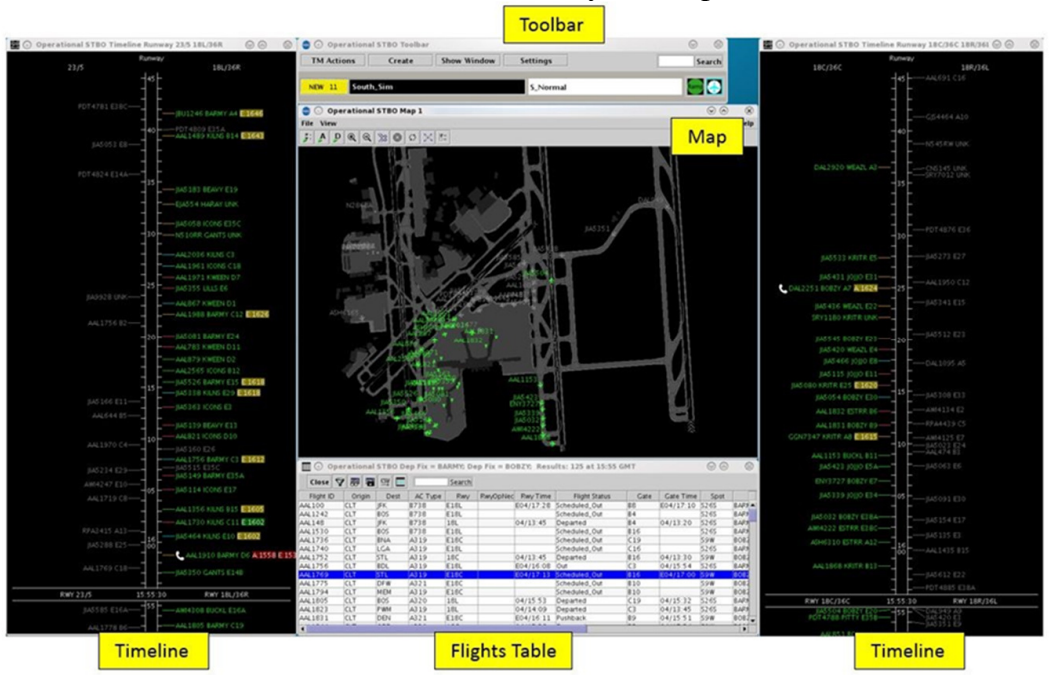

Fig. 1. The Surface Trajectory Based Operations (STBO) Client tool placed in the Tower, TRACON, and Center.

The leader lines of the aircraft are colored to indicate the current location of the aircraft (not visible in Fig. 1), e.g., whether they are at the gate, in the ramp area untracked by 
the surface surveillance system, in the ramp area tracked by the surface surveillance system, or are on the airport surface. The Flights Table provides more detailed information on each flight, and enables sorting on more than 50 variables, such as destination, departure fix, actual take off time (ATOT), estimated time of arrival (ETA), estimated time of departure (ETD), departure gate, airport origin, tail number, Target Movement Area entry Time (TMAT), etc.

Approval Request (APREQ) Electronic Negotiation. The yellow highlights on the right of some of the aircraft datablocks on the Timelines indicate that the aircraft has a "wheels up" time, i.e., are under a Traffic Management Initiative (TMI) involving an APREQ. These aircraft must depart within a certain time limit. Automation is in place such that the Tower TMC can now negotiate this time electronically via the Timelines with the Center TMC instead of making a phone call, as was previously the case. Other types of TMIs are also shown on the timelines, for example EDCTs (Expect Departure Clearance Times) and Miles-in-Trail (MIT) restrictions between departures to a specific departure fix.

Integration of the Map, the Timelines and the Flights Table. Temporarily selecting an aircraft's callsign on the Timeline also highlights its location on the Map and in the Flights Table; all of these STBO Client elements are integrated, enabling one to find out all information on an aircraft quickly by highlighting its callsign on any one element.

Other Tool Integration Elements. The STBO Client can also be used to enter TMI restrictions--such as current APREQs, MITs, and Ground Stops, and to provide additional input about airport operations such as runway closures, airport configuration, runway utilization, etc., that are also disseminated to other ATD2 users.

Similarly, the ramp can also input their decisions or requests (e.g., runway assignment, flight cancellation and ramp closure) through their RTC and RMTC user interfaces. These decisions and requests are in turn displayed on the STBO Client. With the inputs of both the Tower and the Ramp, the ATD2 tools provide a full operational view of the airport at any given time.

\subsection{Ramp Traffic Console (RTC) and Ramp Manager Traffic Console (RMTC) Tools}

Overview. Fig. 2 shows the datablocks of each aircraft parked at the gate and in the ramp area. The blue datablocks indicate aircraft that will depart from the East runway (18L/36R), the brown indicate those that will depart from the West (18C/36C). The arrivals in the ramp area (not yet at the gate) have green datablocks. The three boxes in the right bottom of the display summarize the number of arrivals, eastbound aircraft, and westbound aircraft. The number in the denominator indicates those that have surface metering advisories, i.e., holds at the gate. By clicking on these boxes, one can access the lists of aircraft that fall into these categories.

By right-clicking on a datablock, one can manage the pushback and movement of a flight from the gate to the spot. Options available are Hold, Pushback, Proceed to Gate, Undo Pushback, and Undo Proceed to Gate. 


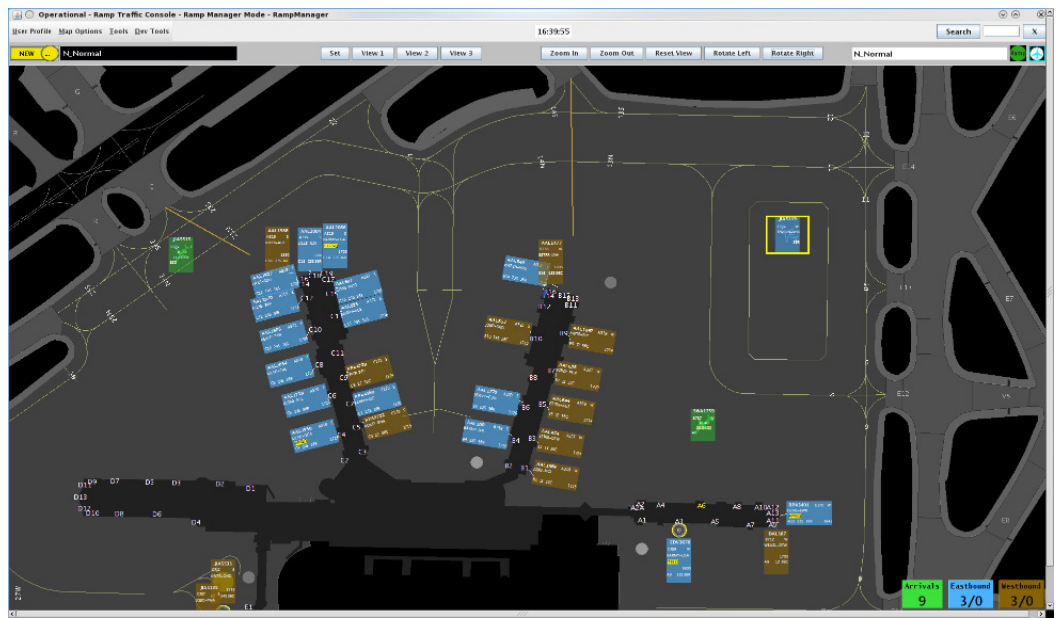

Fig. 2. The Ramp Traffic Console (RTC) placed in the American Airlines Ramp Control Tower at Charlotte-Douglas International Airport.

Flights with an APREQ/CFR and/or EDCT have the relevant information highlighted in yellow, as shown in Fig. 3. A recommended gate hold time is provided to reduce unnecessary time waiting in the runway queue.

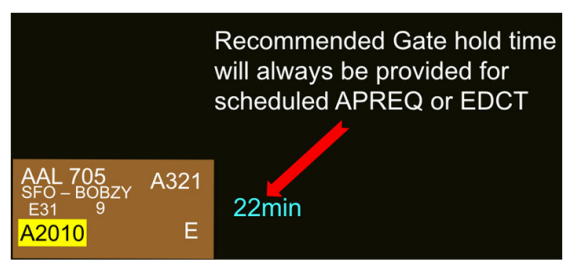

Fig. 3. Close up of flight datablock at gate with APREQ release time highlighted in yellow.

Other aircraft management options available on the RTC update a flight's hardstand, gate, spot, and runway assignments, including changing a runway for operational necessity. All of these updates will appear on both the RTC and STBO displays. The Ramp Manager's version of the RTC, the Ramp Manager Traffic Console (RMTC), differs from the RTC mainly by providing surface metering options for regular aircraft (not in effect in September and November), the operational status of the ramp, and the list of priority flights.

\section{Surveys}

\subsection{Administration}

Online surveys were made available on the primary workstations of the controllers. They were told that the surveys were voluntary, anonymous, and that they could stop at any time in conformance to the requirements of the Institutional Review Board at 
NASA Ames Research Center. Charlotte Airport is noted for aircraft arriving in "banks," or waves of aircraft, with lulls between them when controllers can take breaks. Controllers were asked to take the surveys during these lulls after four of these banks $(2,3,6$, and 7$)$, two occurring in the morning and two in the afternoon.

\subsection{Survey Design}

Helpfulness of ATD2 Tools. How to elicit feedback on the helpfulness of tools posed a problem. The controllers were using their current tools at the same time they were adopting ATD2 tools to use along with their current tools. The introduction of the ATD2 tools occurred at different times depending on the timing of their training. The first step, therefore, was to ascertain the degree to which they were using the ATD2 tools at the same time. This was accomplished by asking the following question to all: "Did you use ATD2 tools to manage your traffic in this bank? There were three options: Yes, I did actively use the ATD2 tools a great deal to make decisions in managing traffic in this bank.

Yes, I did occasionally refer to or use the information provided by ATD2 tools during this bank.

No, I did not use or refer to ATD2 tools for any information in this bank."

The second question asked to all respondents was:

"Please indicate how helpful your tools were in supporting your decisions regarding the following:"

All respondents were asked to rate the degree of helpfulness of their tools on a 1 to 5 Likert-type rating scale depending on the operational duties in their domains. The basic analysis, then, was to compare the ratings on helpfulness of the current tools (no ATD2 tool use) with the use of the new ATD2 tools (plus current tools) on the different items relevant for each type of controller.

Workload and Situational Awareness. In all facilities, controllers were asked to rate their workload using two of the most sensitive NASA TLX rating scales, Mental Demand and Time Pressure [3]. Situational awareness was ascertained by the 3D version of the Situational Awareness Rating Technique (SART), which consists of a score obtained by adding an item rating "your understanding of the traffic situation" to an item rating "the availability of your attentional resources" and subtracting an item rating "demand on your attention" [4].

Acceptability and Efficiency of Airport Operations. Controllers in all facilities were asked to rate the acceptability and efficiency of airport operations that were important in their domain.

Hold times at the Gate for Traffic Management Initiative (TMI) Aircraft. Questions were asked to ramp controllers and managers on how satisfactory the suggested hold times at the gates were for aircraft with APREQs and EDCTs. (Surface metering for all aircraft had not yet begun in September and November of 2017.) 


\section{Results}

\subsection{Response Rates}

The response rates in the facilities varied between $37 \%$ and $75 \%$, as shown in Table 1 . The lower response rates were undoubtedly due to the fact that the controllers and managers were giving up their breaks to take the survey during the lulls between banks.

Table 1. Response rates of personnel in different facilities. (FLM is Front Line Manager)

\begin{tabular}{|l|r|r|r|}
\hline \multicolumn{1}{|c|}{ Respondents } & $\begin{array}{c}\text { Banks } \\
\text { Rated }\end{array}$ & $\begin{array}{c}\text { Possible } \\
\text { Banks to } \\
\text { Rate }\end{array}$ & $\begin{array}{c}\text { Response } \\
\text { Rates }\end{array}$ \\
\hline Ramp controllers Sept. & 51 & 80 & $64 \%$ \\
\hline Ramp controllers Nov. & 72 & 192 & $38 \%$ \\
\hline Ramp managers Sept. & 12 & 16 & $75 \%$ \\
\hline Ramp managers Nov. & 17 & 38 & $45 \%$ \\
\hline Tower TMCs \& FLMs Sept. & 13 & 35 & $37 \%$ \\
\hline TRACON TMCs Sept. & 19 & 40 & $48 \%$ \\
\hline Totals & 184 & 401 & $46 \%$ \\
\hline
\end{tabular}

\section{2 "Helpfulness of Tools" in the Different Facilities}

Tower. Fig. 4 shows that Tower personnel who used current plus ATD2 tools, compared to those who used current tools only, rated their tools as more helpful in supporting decisions regarding "Compliance of aircraft with wheels-up time," i.e., aircraft with EDCTs and APREQs. This was significant at $p=.004$, two tailed t-test, $t(d f 11)=3.6$.

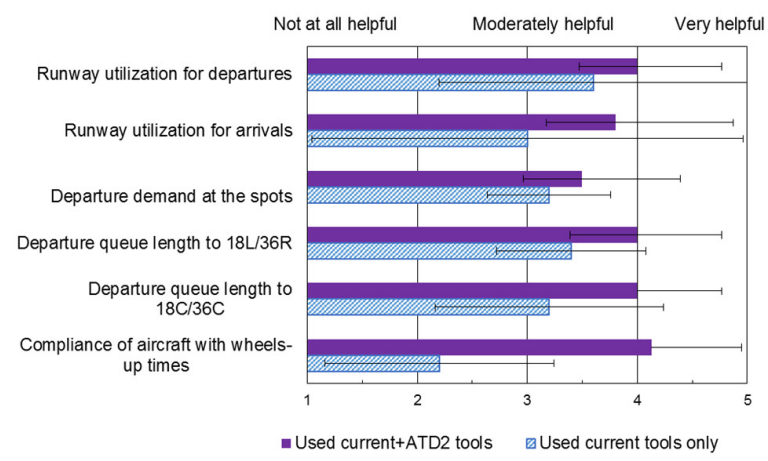

Fig. 4. Tower personnel responses to the question "Please indicate how helpful your tools were in supporting your decisions regarding the following." Error bars are 95\% Confidence Intervals $(C I s)$. (N's varied between 6 and 8 by question for those who used current and ATD2 tools and 5 for those who used current tools only.) 
TRACON. As shown in Fig. 5, the TRACON TMCs who described themselves as using the ATD2 tools actively (as opposed to occasionally or not at all), rated their tools as very helpful, compared to ratings by those who described themselves as using ATD2 tools only occasionally (only one TRACON TMC reported not using the ATD2 tools at all). These differences are all significant beyond $p<.05$, as indicated by the $95 \%$ Confidence Interval error bars. In September, many of the TMCs had just been trained on the tools, and therefore used them only occasionally.

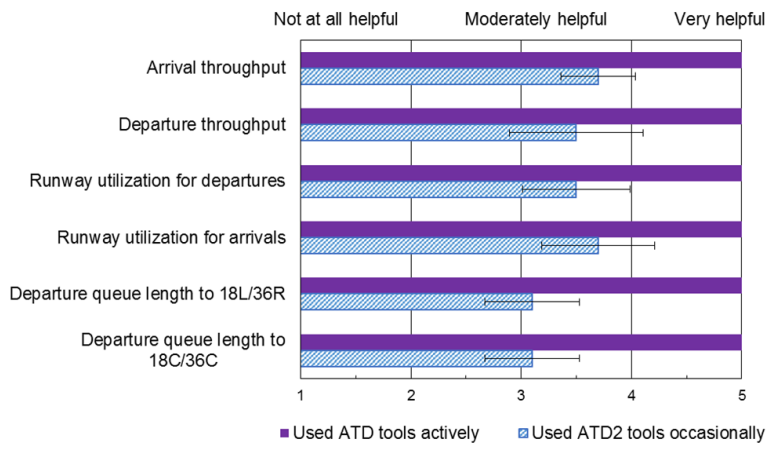

Fig. 5. TRACON TMCs who used the ATD2 tools actively rated the tools as very helpful; those who used them occasionally found the tool moderately helpful. Error bars $=95 \%$ CIs. Ns $=3$ active vs. 14 occasional users.

Ramp Controllers. As can be seen from Fig. 6, in September, the ramp controllers judged their tools more helpful without ATD2 tools regarding decisions on traffic flow.

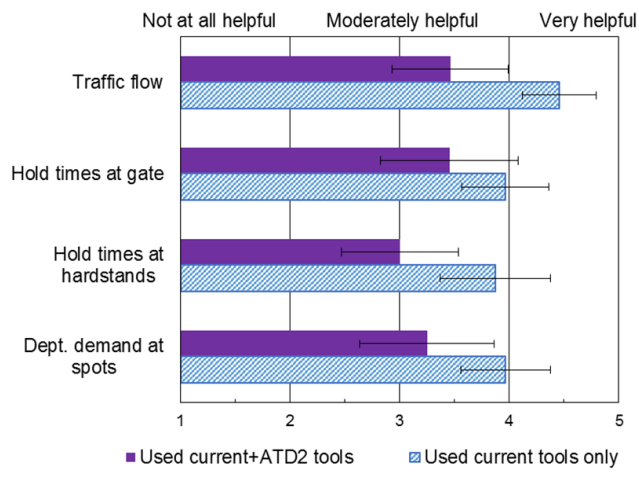

Fig. 6. Ramp controllers' ratings of helpfulness of tools in September, showing current tools more helpful in making decisions about traffic flow than ATD2 tools. Error bars $=95 \% \mathrm{CIs}$. $N \mathrm{~s}=13$ active and occasional users of ATD2 tools and 35 with no ATD2 tool use. Traffic flow significant at $p=.002$, two tailed t-test $t(d f 46),=3.21, N \mathrm{~s}=13$ active $\&$ occasional vs. 35 current tools only. 
In November, however, the ATD2 tools were rated by the ramp controllers as significantly more helpful $(p<.05)$ than they were in September in all areas except holding at the gate, as shown in Fig. 7. In November, there was no statistically significant difference between those who used and didn't use ATD2 tools.

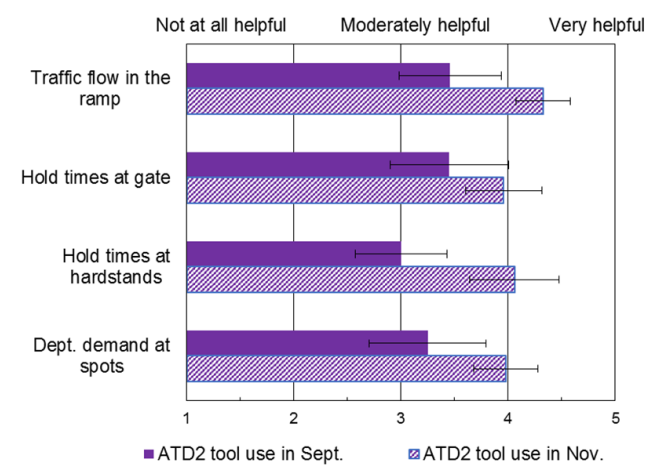

Fig. 7. Ramp controllers' ratings of helpfulness of tools in November, showing ATD2 tool use significantly more helpful in November than in September except for hold times at the gate. Error bars $=95 \%$ CIs. Ns $=11-13$ varying by item in Sept. except 7 for "hold times at hardstands;" 4755 Nov. except 34 for "hold times at hardstands."

Ramp Managers. Very few ramp managers used the ATD2 tools in September; only 2 used them occasionally, 10 did not use them at all. Those using current tools rated them as helpful. In November, tools including ATD2 tools (active and occasional use) were rated as less helpful than current tools had been in Sept on decisions on queue lengths of both runways $(M$ Sept. $=4.9, \mathrm{n} 10 ; M$ Nov. $=3.3, \mathrm{n}=13) . N$ s of banks $=10$ in Sept. (no ATD2 use), length of queues to both runways at, $t(d f 13.3)=3.6, p=.003$.

\subsection{Workload and Situational Awareness}

Overall workload was moderate for all respondents with all tool use. There were no significant differences with tool use. In the Tower, the SART Situational Awareness Measure (3D) indicated higher situational awareness when ATD2 tools were used in the bank, as shown in Fig. 8.

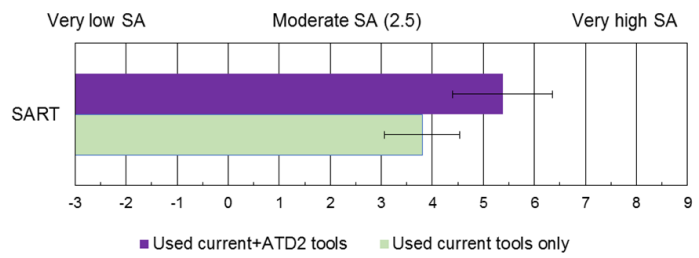

Fig. 8. On the 3D SART measure, Tower controllers who used ATD2 tools indicated that their situational awareness was somewhat higher than those that did not use ATD2 tools. Means were 5.4 and 3.8. $N \mathrm{~s}=8$ banks had some use of ATD2 tools ( 3 active, 5 occasional); 5 did not, $t(d f 11)=2.24, p=.046$. Error bars $=95 \%$ CIs. 
There were no significant differences on SA related to tool use for any of the other respondents except for ramp controllers in September, who scored slightly lower on SA when using the ATD2 tools, Means 3.3 and 4.3, Ns $=11$ and 32, $t(\operatorname{df} 41)=2.0, p=.03$. In November, the reverse was true, although the difference did not quite reach the level of significance. The means were 4.7 with and 3.7 without ATD2 use, two-tailed $t(d f$ $20.2)=1.9, p=.07$, equal variances not assumed.

\subsection{Acceptability}

On the whole, the findings on acceptability were similar to the findings on helpfulness of tools. For example, in the Tower, the wheels-up time for aircraft with TMIs were seen as more acceptable with ATD2 use at $p=.02$. In the TRACON, the length of the queue at $18 \mathrm{~L} / 36 \mathrm{R}$ was seen as more acceptable with active vs. occasional use at $p=$ .04 .

And again, in the ramp in September, ATD2 tool use was associated with less acceptable hold times at the gate $(p=.06)$ and at the hardstands $(p=.008)$ as shown in Fig. 9.

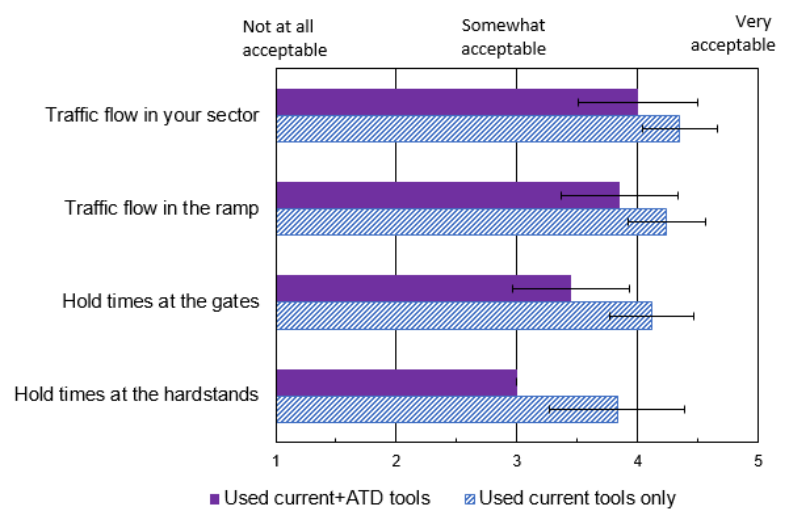

Fig. 9. Rated acceptability of ramp operations by ramp controllers in September showed less acceptable hold times at the gates and hardstands when using ATD2 tools. Error bars are 95\% CIs. Ns $=13$ (used ATD2 tools) and 37 (did not use) except for hold times at hardstand at 5 and 23.

However, in November, this trend was reversed such that all measures were significantly equal or better with ATD2 use except hold times at the gate, as shown in Fig. 10. 


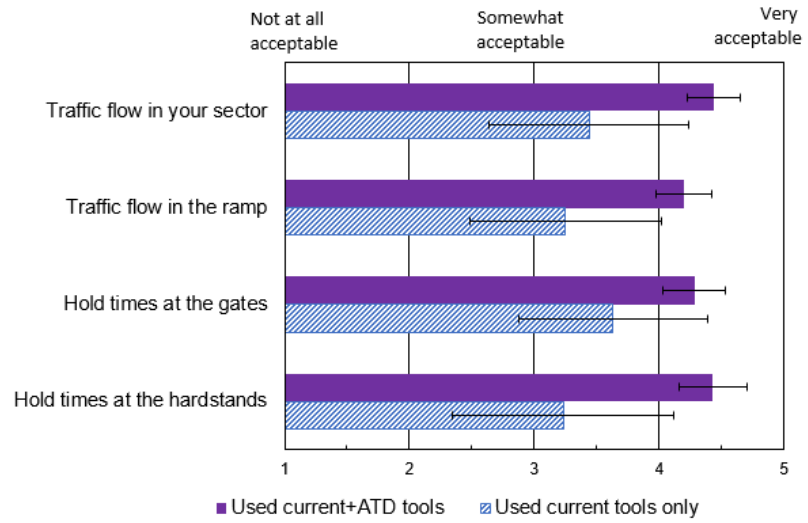

Fig. 10. Ramp controllers' ratings reversed from September's to indicate more acceptable operations in November associated with ATD2 tool use. All measures significantly equal or better with ATD2 use except hold time at gate. Traffic flow in sector $p=.03$, in ramp $p=.03$, and hold times at hardstand $p=.02 . N \mathrm{~s}=13-16$ current tools only, 35-55 ATD2 tool use. Error bars are $95 \%$ CIs.

For ramp managers, all measures were acceptable both in September and November regardless of tool usage. However, runway queue lengths were seen as less acceptable in November with ATD2 use than in September with no ATD2 use, Means 3.7, Sept., and 4.7, Nov. ( $N$ s $=10$ in Sept. and 16 in Nov., $p<.05$.).

\subsection{Hold Times at the Gate for Traffic Management Initiative (TMI) Aircraft}

Ramp controllers in September and November thought that the TMI gate hold times recommended to reduce queue length at the runways were about right. They almost never held aircraft after they were advised to push the aircraft off the gate. If they did, it was generally because other aircraft were blocking the alley, or rarely, to release another TMI flight. They would not have changed the recommended gate hold times.

The ramp managers in November rated the TMI gate hold times as being on the short side. Many (38\% or $6 / 16)$ would have changed the TMI hold times, commenting that the departure queue lengths needed to be taken more into account.

\section{Summary and Discussion}

The implementation of the data exchange and integration elements into the CharlotteDouglas Airport facilities is an important and necessary step in achieving the ultimate goals of ATD2: the integration of the Arrival and Departure Surface (IADS) traffic management capabilities. One of the first human factor assessment queries was whether the additional data exchange elements increased workload and this did not appear to have happened. Situational awareness was improved for Tower with ATD2 use and in November, for ramp controllers. 
The surveys elicited positive results from Tower and TRACON personnel in terms of the helpfulness of ATD2 tools in conveying the initial data exchange elements. Specifically, in the Tower, the ATD2 tools seemed to be helpful in supporting decisions regarding "Compliance of aircraft with wheels-up time." This is congruent with the Tower TMCs' new ability to monitor TMI aircraft on the STBO Client Timelines and to negotiate new times electronically with the Center TMCs if it appears that aircraft are not going to make their originally scheduled times.

In the TRACON, those who used the ATD2 tools actively found their tools significantly more helpful than those who used them only occasionally. In the TRACON, even the ramp tool was helpful. On the survey, TRACON TMCs were asked the question, "If you changed the runway assignments for the arriving aircraft, what was the reason?" The reason most frequently checked was "to remove load on runway to allow reduction of the departure queue." Current tools indicate the number of aircraft going to the different runways only on the Airport Movement Area (AMA). Although this enables the TMCs to predict runway queue length in the near future, the RTC shows the number of aircraft going to the different runways still at the gate and taxiing in the ramp, allowing the TMCs to predict runway queue length even further in the future.

In the ramp, when ATD2 tools use was just beginning in September, those using their current tools rated them as more helpful in accomplishing their duties than those who used the current tools along with ATD2 tools. In November, just a few months later, those ratings were reversed, with those using the ATD2 tools rating their tools as significantly more helpful in supporting decisions than those using the current tools in September. A similar reversal took place regarding ratings on the acceptability of operations in the ramp, with the November ratings favoring ATD2 use.

In sum, although some parameters need to be adjusted, as indicated by the ramp managers' ratings in November on queue length and gate hold times, the integration of the data exchange elements in Phase 1 of the ATD2 project appears to be successful in terms of human factors metrics. It is probable that continued use of these tools will only improve these metrics, as was demonstrated by the increase in favorable ramp controllers' responses in the two-month period from September to November 2017.

\section{References}

1. NASA Airspace Technology Demonstration 2 (ATD2): https://www.aviationsystemsdivision.arc.nasa.gov/research/atd2/index.shtml

2. FAA Air Traffic Organization Surface Operations Office, U.S. Airport Surface Collaborative Decision Making (CDM) Concept of Operations (ConOps) in the Near-Term (2012), https://faaco.faa.gov/index.cfm/attachment/download/33926

3. Hart, S. G., \& Staveland, L. E. Development of a NASA-TLX (Task Load Index): Results of Empirical and Theoretical Research. In: Hancock, P.S., Meshkati, N., Human Mental Workload, pp. 139--183, Elsevier Science Publishers B. V., Amsterdam (1988)

4. Taylor, R.M.: Situational Awareness Rating Technique (SART): The Development of a Tool for Aircrew Systems Design. In: Proceedings of the AGARD AMP Symposium on Situational Awareness in Aerospace Operations, CP478. Seuilly-sur Seine: NATO AGARD (1989) 